\section{MS20-01 A structural perception about intrinsic point defects in kesterite type compound semiconductors}

\author{
Susan Schorr $^{1}$
}

\section{Helmholtz-Zentrum Berlin für Materialien und Energie}

email: susan.schorr@helmholtz-berlin.de

The understanding of the interplay between structural, chemical and electronic properties of kesterite type compound semiconductors, applied as absorber materials in photovoltaic devices, can give crucial informations aiding the continuous improvement of device efficiencies. Various reasons are discussed about efficiency losses in the bulk material of kesterite type based solar cells. These absorber layers, which are $\mathrm{Cu}_{2} \mathrm{ZnSn}(\mathrm{S}, \mathrm{Se})_{4}$, exhibit in general a off-stoichiometric composition (Cu-poor, Zn-rich). The presence of secondary phases and deep defect levels caused by intrinsic point defects are efficiency limiting factors. The present study reports a detailed crystallographic investigation about cationic point defects in off-stoichiometric $\mathrm{Cu}_{2} \mathrm{ZnSnS}_{4}$ and $\mathrm{Cu}_{2} \mathrm{ZnSnSe}{ }_{4}$. Both compounds crystallize in the kesterite type structure (space group I-4). The basis of the systematic study are series of powder samples synthesized by solid state reaction. Their chemical composition has been determined by WDX spectroscopy (electron microprobe analysis). Due to the isoelectronic character of $\mathrm{Cu}^{+}$and $\mathrm{Zn}^{2+}$ it is necessary to apply neutron powder diffraction to investigate the distribution of the three cations on the four cationic sites of the kesterite type structure. Rietveld refinements and the average neutron scattering length analysis method have been used to evaluate the neutron diffraction data. The kesterite type structure can be described by a stacking sequence of cation layerd $\mathrm{Cu} / \mathrm{Sn}-\mathrm{Cu} / \mathrm{Zn}-\mathrm{Sn} / \mathrm{Cu}-\mathrm{Cu} / \mathrm{Zn}-\mathrm{Cu} / \mathrm{Sn}$ perpendicular to the crystallographic z-direction. An off-stoichiometric composition, for instance $\mathrm{Cu}$-poor, originates from the prospensity of the structure to stabilize copper vacancies, the charge balancees beeing commonly insured by appropriate substitutions on the cationic sites. If the oxidation states of the cations and anions are retained going from stoichiometric to an off-stoichiometric composition, a number of specific substitutions can be envisioned to account for the charge balance in the off-stoichiometric material. These substitutions lead to the formationof point defects in the crystal structure. The presented study shows, that the point defects, expected by this model, are found experimentally in the synthesized compounds. This demonstrates the ability of the kesterite type phase to tolerate large deviations from stoichiometric composition without collaps of the structure. 